\title{
Text Summarization Challenge 2
}

\section{Text summarization evaluation at NTCIR Workshop 3}

\author{
Manabu Okumura \\ Tokyo Institute of Technology \\ oku@pi.titech.ac.jp
}

\author{
Takahiro Fukusima \\ Otemon Gakuin University \\ fukusima@res.otemon.ac.jp
}

\author{
Hidetsugu Nanba \\ Hiroshima City University \\ nanba@its.hiroshima-cu.ac.jp
}

\begin{abstract}
We describe the outline of Text Summarization Challenge 2 (TSC2 hereafter), a sequel text summarization evaluation conducted as one of the tasks at the NTCIR Workshop 3. First, we describe briefly the previous evaluation, Text Summarization Challenge (TSC1) as introduction to TSC2. Then we explain TSC2 including the participants, the two tasks in TSC2, data used, evaluation methods for each task, and brief report on the results.
\end{abstract}

Keywords: automatic text summarization, summarization evaluation

\section{Introduction}

As research on automatic text summarization is being a hot topic in NLP, we also see the needs to discuss and clarify the issues on how to evaluate text summarization systems. SUMMAC in May 1998 as a part of TIPSTER (Phase III) project ([1], [2]) and Document Understanding Conference (DUC) ([3]) in the United States show the need and importance of the evaluation for text summarization.

In Japan, Text Summarization Challenge (TSC1), a text summarization evaluation, the first of its kind, was conducted in the years of 1999 to 2000 as a part of the NTCIR Workshop 2. It was realized in order for the researchers in the field to collect and share text data for summarization, and to make clearer the issues of evaluation measures for summarization of Japanese texts ([4],[5],[6]). TSC1 used newspaper articles and had two tasks for a set of single articles with intrinsic and extrinsic evaluations. The first task (task A) was to produce summaries (extracts and free summaries) for intrinsic evaluations. We used recall, precision and Fmeasure for the evaluation of the extracts, and contentbased as well as subjective methods for the evaluation of the free summaries.

The summarization rates for task A were as follows:
$10,30,50 \%$ for extracts and $20,40 \%$ for free summaries.

The second task (task B) was to produce summaries for information retrieval (relevance judgment) task. The measures for evaluation were recall, precision and Fmeasure to indicate the accuracy of the task, as well as the time to indicate how long it takes to carry out the task.

We also prepared human-produced summaries including key data for the evaluation. In terms of genre, we used editorials and business news articles at TSC1's dryrun, and editorials and articles on social issues at the formal run evaluation.

As sharable data, we had summaries for 180 newspaper articles by spring 2001. For each article, we had the following seven types of summaries: important sentences $(10,30,50 \%)$, important parts specified (20, $40 \%)$, and free summaries $(20,40 \%)$.

In comparison, TSC2 uses newspaper articles and has two tasks (single- and multi-document summarization) for two types of intrinsic evaluations. In the following sections, we describe TSC2 in detail.

\section{Two Tasks in TSC2 and its Schedule}

TSC2 has two tasks. They are single document summarization (task A) and multi-document summarization (task B).

Task A: We ask the participants to produce summaries in plain text to be compared with humanprepared summaries from single documents. Summarization rate is a rate between the number of characters in the summary and the total number of characters in the original article. The rates are about $20 \%$ and $40 \%$. This task is the same as task A-2 in TSC1.

Task B: In this task, more than two (multiple) documents are summarized for the task. Given a set of documents, which has been gathered for a pre-defined topic, the participants produce summaries of the set in plain text format. The information that was used to produce the document set, such as queries, as well as 
summarization lengths are given to the participants. Two summarization lengths are specified, short and long summaries for one set of documents.

The schedule of evaluations at TSC2 was as follows: dryrun was conducted in December 2001 and formal run was in May 2002. The final evaluation results were reported to the participants by early July 2002.

\section{Data Used for TSC2}

We use newspaper articles from the Mainichi newspaper database of 1998, 1999. As key data (human prepared summaries), we prepare the following types of summaries.

\section{Extract-type summaries:}

We asked captioners who are well experienced in summarization to select important sentences from each article. The summarization rates are $10 \%, 30 \%$, and $50 \%$.

Abstract-type summaries:

We asked the captioners to summarize the original articles in two ways. The first is to choose important parts of the sentences recognized important in extract-type summaries (abstract-type type1). The second is to summarize the original articles "freely" without worrying about sentence boundaries, trying to obtain the main idea of the articles (abstract-type type2). Both types of abstract-type summaries are used for task A. The summarization rates are $20 \%$ and $40 \%$.

Both extract-type and abstract-type summaries are summaries from single articles.

Summaries from more than two articles:

Given a set of newspaper articles that has been selected based on a certain topic, the captioners produced free summaries (short and long summaries) for the set. Topics are various, from kidnapping case to $\mathrm{Y} 2 \mathrm{~K}$ problem.

\section{Evaluation Methods for each task}

We use summaries prepared by human as key data for evaluation. The same two intrinsic evaluation methods are used for both tasks. They are evaluation by ranking summaries and by measuring the degree of revisions. Here are the details of the two methods. We use 30 articles for task A and 30 sets of documents (30 topics) for task $\mathrm{B}$ at formal run evaluation. Unfortunately, due to the limitation of the budget, only an evaluator evaluates a system's result for an article(or a set).

\subsection{Evaluation by ranking}

This is basically the same as the evaluation method used for TSC1 task A-2 (subjective evaluation). We ask human judges, who are experienced in producing summaries, to evaluate and rank the system summaries in terms of two points of views.

1. Content: How much the system summary covers the important content of the original article.

2. Readability: How readable the system summary is. The judges are given 4 types of summaries to be evaluated and rank them in 1 to 4 scale ( 1 is the best, 2 for the second, 3 for the third best, and 4 for the worst).

For task A, the first two types are human-produced abstract-type type 1 and type 2 summaries. The third is system results, and the fourth is summaries produced by lead method.

For task B, the first is human-produced free summaries of the given set of documents, and the second is system results. The third is the results of the baseline system based on lead method where the first sentence of each document is used. The fourth is the results of the benchmark system using Stein method ([7]) whose procedure is as follows:

1. Produce a summary for each document.

2. Group the summaries into several clusters. The number of clusters is adjusted to be less than the half of the number of the documents.

3. Choose the most representative summary as the summary of the cluster.

4. Compute the similarity among the clusters and output the representative summaries in such order that the similarity of neighboring summaries is high.

\subsection{Evaluation by revision}

It is a newly introduced evaluation method in TSC2 to evaluate the summaries by measuring the degree of revision to system results. The judges read the original documents and revise the system summaries in terms of the content and readability. The revisions are made by one of three editing operations (insertion, deletion, replacement). The degree of the revision is computed based on the number of the operations and the number of revised characters. The revisers could be completely free in what they did, though they were instructed to do minimum revision.

As baseline for task A, lead-method results are used. As reference for task A, human produced summaries (abstract type1 and abstract type 2) are used. And as baseline, reference, and benchmark for task B, leadmethod results, human produced summaries that are different from the key data, and the results based on the Stein method are used respectively. 
When more than half of the document needs to be revised, the judges can 'give up' revising the document.

\section{Participants}

We had 4 participating systems for Task A, and 5 systems for Task B at dryrun. We have 8 participating systems for Task A and 9 systems for Task B at formal run. As group, we had 8 participating groups, which are all Japanese, of universities, governmental research institute or companies in Japan. Table 1 shows the breakdown of the groups.

\begin{tabular}{|l|l|}
\hline University & 6 \\
\hline $\begin{array}{l}\text { Governmental } \\
\text { research institute }\end{array}$ & 1 \\
\hline Company & 2 \\
\hline
\end{tabular}

Table 1 Breakdown of Participants (Please note that one group consists of a company and a university.)

\section{Results}

\subsection{Results of Evaluation by ranking}

Table 2 shows the result of evaluation by ranking for task $A$ and Table 3 shows the result of evaluation by ranking for task B. Each score is the average of the scores for 30 articles for task A, and 30 topics for task B at formal run.

\begin{tabular}{|c|c|c|c|c|}
\hline $\begin{array}{c}\text { System } \\
\text { No }\end{array}$ & $\begin{array}{c}\text { Content } \\
20 \%\end{array}$ & $\begin{array}{c}\text { Read- } \\
\text { ability } \\
20 \%\end{array}$ & $\begin{array}{c}\text { Content } \\
40 \%\end{array}$ & $\begin{array}{c}\text { Read- } \\
\text { ability } \\
40 \%\end{array}$ \\
\hline F0101 & 2.53 & 2.87 & 2.60 & 2.77 \\
\hline F0102 & 2.67 & 2.97 & 2.50 & 2.77 \\
\hline F0103 & 2.80 & 2.93 & 2.90 & 2.90 \\
\hline F0104 & 2.77 & 2.73 & 2.80 & 2.90 \\
\hline F0105 & 2.70 & 2.73 & 2.60 & 2.77 \\
\hline F0106 & 2.73 & 2.57 & 2.63 & 2.67 \\
\hline F0107 & 2.70 & 2.60 & 2.50 & 2.53 \\
\hline F0108 & 2.40 & 2.83 & 2.60 & 2.77 \\
\hline TF & 3.30 & 3.30 & 3.20 & 3.10 \\
\hline Human & 2.33 & 2.20 & 2.10 & 2.03 \\
\hline
\end{tabular}

Table 2 Ranking evaluation (task A)

In Tables 2 and 3, F01* and F02* are labels for the different systems involved, respectively. In Table 2, ' $\mathrm{TF}$ ' indicates a baseline system based on termfrequency method, and 'Human' indicates human- produced summaries that are different from the key data used in ranking judgement.

In Table 3, 'Human' indicates human-produced summaries that are different from the key data used in ranking judgement.

\begin{tabular}{|c|c|c|c|c|}
\hline System No & $\begin{array}{c}\text { Content } \\
\text { Short }\end{array}$ & $\begin{array}{c}\text { Read- } \\
\text { ability } \\
\text { Short }\end{array}$ & $\begin{array}{c}\text { Content } \\
\text { Long }\end{array}$ & $\begin{array}{c}\text { Read- } \\
\text { ability } \\
\text { Long }\end{array}$ \\
\hline F0201 & 2.70 & 3.17 & 2.50 & 3.23 \\
\hline F0202 & 2.73 & 2.70 & 2.77 & 2.93 \\
\hline F0203 & 2.60 & 2.33 & 2.97 & 3.03 \\
\hline F0204 & 2.63 & 2.90 & 2.80 & 3.03 \\
\hline F0205 & 2.53 & 3.10 & 2.73 & 3.30 \\
\hline F0206 & 3.20 & 3.00 & 3.47 & 3.30 \\
\hline F0207 & 2.40 & 2.87 & 2.63 & 3.27 \\
\hline F0208 & 2.93 & 2.70 & 2.53 & 2.80 \\
\hline F0209 & 2.83 & 2.73 & 2.53 & 2.87 \\
\hline Human & 2.00 & 2.17 & 1.83 & 2.33 \\
\hline
\end{tabular}

Table 3 Ranking evaluation (task B)

In Appendix A, we also show tables giving the fraction of time that each system beats the baseline, one human summary, or two human summaries for task A. In Appendix B, we show tables giving the fraction of time that each system beats the baseline, the benchmark, or human summary for task B.

\begin{tabular}{|c|c|c|c|c|}
\hline & $\begin{array}{c}\text { Content } \\
20 \%\end{array}$ & $\begin{array}{c}\text { Read- } \\
\text { ability } \\
20 \%\end{array}$ & $\begin{array}{c}\text { Content } \\
40 \%\end{array}$ & $\begin{array}{c}\text { Read- } \\
\text { ability } \\
40 \%\end{array}$ \\
\hline $\begin{array}{c}\text { Human } \\
\text { type 1) }\end{array}$ & 1.58 & 1.61 & 1.67 & 1.69 \\
\hline $\begin{array}{c}\text { Human } \\
\text { type 2) }\end{array}$ & 1.50 & 1.57 & 1.42 & 1.55 \\
\hline $\begin{array}{c}\text { Baseline } \\
\text { (Lead) }\end{array}$ & 3.80 & 3.60 & 3.83 & 3.55 \\
\hline
\end{tabular}

Table 4 Ranking evaluation (task A, human and baseline)

\begin{tabular}{|c|c|c|c|c|}
\hline & $\begin{array}{c}\text { Content } \\
\text { Short }\end{array}$ & $\begin{array}{c}\text { Read- } \\
\text { ability } \\
\text { Short }\end{array}$ & $\begin{array}{c}\text { Content } \\
\text { Long }\end{array}$ & $\begin{array}{c}\text { Read- } \\
\text { ability } \\
\text { Long }\end{array}$ \\
\hline $\begin{array}{c}\text { Human } \\
\text { (type 2) }\end{array}$ & 1.65 & 2.38 & 1.82 & 2.38 \\
\hline $\begin{array}{c}\text { Baseline } \\
\text { (Lead) }\end{array}$ & 2.80 & 2.20 & 2.70 & 2.22 \\
\hline $\begin{array}{c}\text { Benchmark } \\
\text { (Stein) }\end{array}$ & 2.48 & 2.00 & 2.50 & 1.99 \\
\hline
\end{tabular}

Table 5 Ranking evaluation (task B, human, baseline, and benchmark) 
In comparison with the system results (Table 2 and Table 3), the scores for the human summaries, the baseline systems, and the benchmark system(the summaries to be compared) are shown in Table 4 and Table 5.

\subsection{Results of Evaluation by revision}

Table 6 shows the result of evaluation by revision for task A at rate $40 \%$, and Table 7 shows the result of evaluation by revision for task $\mathrm{A}$ at rate $20 \%$. Table 8 shows the result of evaluation by revision for task B long, and Table 9 shows the result of evaluation by revision for task B short. All the tables show the evaluation results in terms of average number of revisions (editing operations) per document.

\begin{tabular}{|l|c|c|c|c|c|c|}
\hline \multirow{2}{*}{ System } & \multicolumn{2}{|l|}{ Deletion } & \multicolumn{2}{l|}{ Insertion } & \multicolumn{2}{l|}{ Replacement } \\
\cline { 2 - 7 } & UIM & RD & IM & RD & C & RD \\
\hline F0101 & 2.0 & 0.1 & 1.5 & 0.4 & 0.5 & 0.7 \\
\hline F0102 & 1.6 & 0.4 & 1.5 & 0.4 & 0.4 & 0.8 \\
\hline F0103 & 2.3 & 0.2 & 2.4 & 0.2 & 0.4 & 0.5 \\
\hline F0104 & 2.4 & 0.4 & 2.7 & 0.5 & 0.4 & 0.5 \\
\hline F0105 & 2.0 & 0.3 & 1.7 & 0.1 & 0.7 & 0.7 \\
\hline F0106 & 2.8 & 0.2 & 2.3 & 0.4 & 0.3 & 0.6 \\
\hline F0107 & 2.5 & 0.6 & 1.8 & 0.2 & 0.1 & 0.5 \\
\hline F0108 & 2.0 & 0.4 & 2.4 & 0.1 & 0.4 & 0.6 \\
\hline ld & 2.9 & 0.1 & 0.7 & 0.1 & 0.4 & 0.1 \\
\hline free & 0.4 & 0.4 & 1.2 & 0.4 & 0.1 & 0.3 \\
\hline part & 0.7 & 0.6 & 0.9 & 0.3 & 0.1 & 0.4 \\
\hline edit & 0.3 & 0.1 & 0.4 & 0.3 & 0.1 & 0.2 \\
\hline ALL & 1.9 & 0.3 & 1.8 & 0.3 & 0.3 & 0.5 \\
\hline
\end{tabular}

Table 6 Evaluation by revision (task A 40\%)

Please note that UIM stands for unimportant, RD for readability, IM for important, $\mathrm{C}$ for content in Tables 6 to 9. They mean the reason for the operations, e.g. 'unimportant' is for deletion operation due to the part judged to be unimportant, and 'content' is for replacement operation due to excess and deficiency of content.

In Table 6 and Table 7, 'ld' means a baseline system using lead method, 'free' is free summaries produced by human (abstract type 2), and 'part' is human-produced (abstract type1) summaries, and these three are baseline and reference scores for task A.

\begin{tabular}{|l|c|c|c|c|c|c|}
\hline \multirow{2}{*}{ System } & \multicolumn{2}{|l|}{ Deletion } & \multicolumn{2}{l|}{ Insertion } & \multicolumn{2}{l|}{ Replacement } \\
\cline { 2 - 7 } & UIM & RD & IM & RD & C & RD \\
\hline F0101 & 1.4 & 0.4 & 1.3 & 0.2 & 0.5 & 0.3 \\
\hline F0102 & 1.2 & 0.4 & 1.0 & 0.0 & 0.4 & 0.5 \\
\hline F0103 & 0.8 & 0.1 & 1.2 & 0.0 & 0.2 & 0.1 \\
\hline F0104 & 0.8 & 0.1 & 1.2 & 0.1 & 0.1 & 0.2 \\
\hline F0105 & 1.2 & 0.1 & 0.7 & 0.0 & 0.4 & 0.2 \\
\hline F0106 & 2.1 & 0.2 & 1.7 & 0.1 & 0.1 & 0.2 \\
\hline F0107 & 0.8 & 0.6 & 0.9 & 0.1 & 0.2 & 0.1 \\
\hline F0108 & 1.4 & 0.1 & 1.1 & 0.1 & 0.2 & 0.6 \\
\hline ld & 1.9 & 0.1 & 1.3 & 0.0 & 0.0 & 0.0 \\
\hline free & 0.6 & 0.4 & 1.1 & 0.1 & 0.2 & 0.1 \\
\hline part & 0.7 & 0.3 & 1.1 & 0.1 & 0.1 & 0.2 \\
\hline edit & 0.2 & 0.1 & 0.5 & 0.1 & 0.2 & 0.2 \\
\hline ALL & 1.1 & 0.3 & 1.1 & 0.1 & 0.2 & 0.3 \\
\hline
\end{tabular}

Table 7 Evaluation by revision (task A 20\%)

\begin{tabular}{|l|c|c|c|c|c|c|}
\hline \multirow{2}{*}{ System } & \multicolumn{2}{|l|}{ Deletion } & \multicolumn{2}{l|}{ Insertion } & \multicolumn{2}{l|}{ Replacement } \\
\cline { 2 - 7 } & UIM & RD & IM & RD & C & RD \\
\hline F0201 & 3.8 & 0.7 & 7.2 & 1.4 & 1.1 & 0.9 \\
\hline F0202 & 5.2 & 0.6 & 3.5 & 0.4 & 0.7 & 0.5 \\
\hline F0203 & 5.1 & 0.6 & 3.8 & 0.5 & 0.9 & 0.6 \\
\hline F0204 & 4.2 & 0.6 & 3.4 & 0.7 & 1.4 & 0.7 \\
\hline F0205 & 8.1 & 0.6 & 5.4 & 1.7 & 3.0 & 1.3 \\
\hline F0206 & 3.2 & 0.2 & 4.7 & 0.7 & 0.8 & 0.6 \\
\hline F0207 & 7.0 & 1.1 & 4.1 & 1.1 & 1.1 & 1.1 \\
\hline F0208 & 4.8 & 0.7 & 4.0 & 0.4 & 0.8 & 0.9 \\
\hline F0209 & 4.6 & 0.5 & 3.9 & 0.5 & 0.5 & 0.5 \\
\hline human & 3.0 & 0.9 & 3.4 & 7.8 & 1.0 & 1.2 \\
\hline ld & 5.7 & 0.9 & 2.9 & 0.4 & 0.7 & 0.5 \\
\hline stein & 4.0 & 0.5 & 2.2 & 0.3 & 0.8 & 0.5 \\
\hline edit & 3.0 & 1.2 & 2.9 & 0.7 & 0.7 & 1.1 \\
\hline ALL & 4.9 & 0.7 & 4.0 & 1.3 & 1.1 & 0.8 \\
\hline
\end{tabular}

Table 8 Evaluation by revision (task B long)

In Table 8 and Table 9, 'human' means humanproduced summaries which are different from the key data, and 'ld' means a baseline system using lead method, 'stein' means a benchmark system using Stein method, and these three are baseline, reference, and benchmark scores for task B.

To determine the plausibility of the judges' revision, the revised summaries were again evaluated with the evaluation methods in section 5. In Tables 6 to 9, 'edit' means the evaluation results for the revised summaries.

We also measure as degree of revision the number of revised characters for the three editing operations, and the number of documents that are given up revising by 
the judges. Please look at the detailed data at NTCIR Workshop 3 data booklet.

\begin{tabular}{|l|c|c|c|c|c|c|}
\hline \multirow{2}{*}{ System } & \multicolumn{2}{|l|}{ Deletion } & \multicolumn{2}{l|}{ Insertion } & \multicolumn{2}{l|}{ Replacement } \\
\cline { 2 - 7 } & $\begin{array}{c}\text { UI } \\
\text { M }\end{array}$ & RD & IM & RD & C & RD \\
\hline F0201 & 3.5 & 0.5 & 4.3 & 0.8 & 1.1 & 0.7 \\
\hline F0202 & 3.5 & 0.4 & 2.4 & 0.2 & 0.7 & 0.2 \\
\hline F0203 & 3.6 & 0.3 & 2.8 & 0.2 & 0.5 & 0.4 \\
\hline F0204 & 2.7 & 0.5 & 2.3 & 0.2 & 1.2 & 0.7 \\
\hline F0205 & 5.5 & 0.4 & 2.5 & 0.8 & 2.0 & 0.7 \\
\hline F0206 & 2.0 & 0.4 & 3.4 & 0.6 & 0.4 & 0.4 \\
\hline F0207 & 3.5 & 0.4 & 2.7 & 0.3 & 0.6 & 0.6 \\
\hline F0208 & 2.4 & 0.5 & 2.3 & 0.4 & 0.2 & 0.3 \\
\hline F0209 & 2.5 & 0.5 & 2.2 & 0.2 & 0.3 & 0.4 \\
\hline human & 1.9 & 0.8 & 2.4 & 2.0 & 0.9 & 0.7 \\
\hline ld & 2.8 & 0.7 & 2.4 & 0.2 & 0.5 & 0.4 \\
\hline stein & 3.0 & 0.3 & 1.8 & 0.2 & 0.4 & 0.3 \\
\hline edit & 2.2 & 0.8 & 2.5 & 0.6 & 1.0 & 1.2 \\
\hline ALL & 3.1 & 0.5 & 2.6 & 0.5 & 0.7 & 0.5 \\
\hline
\end{tabular}

Table 9 Evaluation by revision (task B short)

\section{Discussion}

\subsection{Discussion for Evaluation by ranking}

We here further look into how the participating systems perform by analysing the ranking results in terms of differences in scores for content and those for readability.

First, consider task A. Figure 1 shows the differences in scores for content and readability for each system. 'C20-R20' means the score for content $20 \%$ minus the score for readability $20 \%$. 'C40-R40' means the score for content $40 \%$ minus the score for readability $40 \%$.

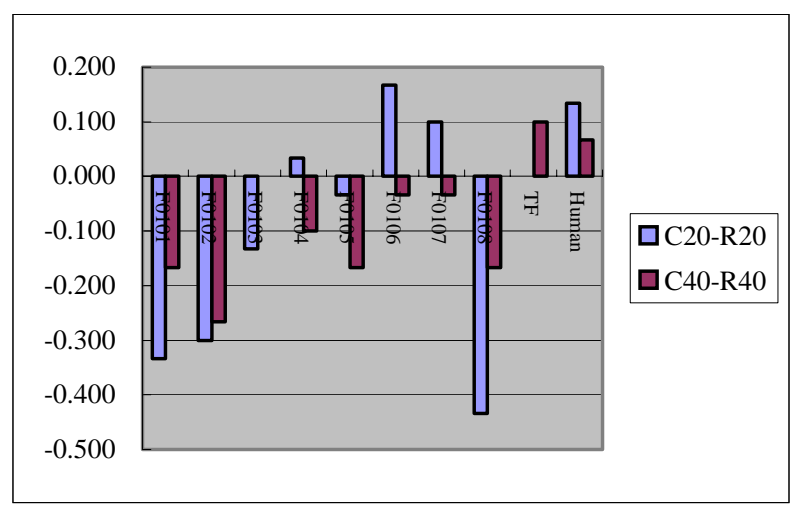

Figure 1 Score difference between Content and Readability (Task A)
Figure 1 indicates how much the scores for content and readability vary for the summaries of the same summarization rate. It shows that the readability scores tend to be higher than those for content, and it is especially clearer for $40 \%$ summarization.

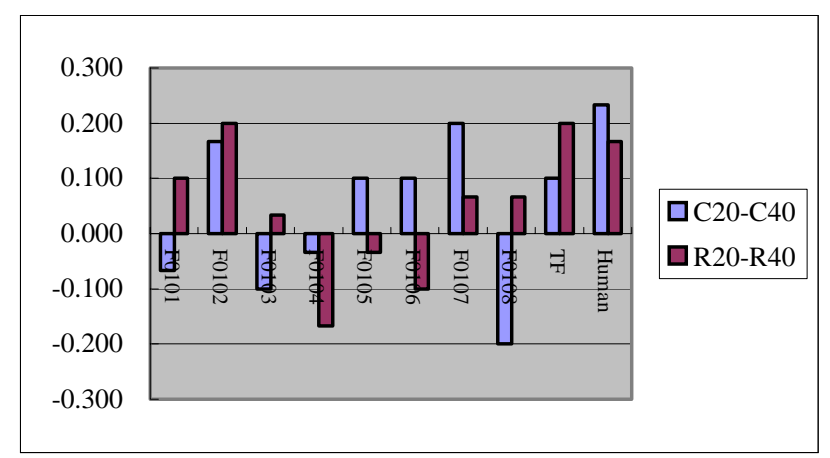

Figure 2 Score difference between $20 \%$ and $40 \%$ summarizations (Task A)

Figure 2 shows the differences in scores for the different summarization rates, i.e. $20 \%$ and $40 \%$ of task A. 'C20-C40' means the score for content $20 \%$ minus the score for content $40 \%$. 'R20-R40' 'means the score for readability $20 \%$ minus the score for readability $40 \%$.

Figure 2 tells us that the ranking scores for $20 \%$ summarization tend to be higher than those for $40 \%$, and this is true with the baseline system and human summaries as well.

Second, consider task B. Figure 3 shows the differences in scores for content and readability for each system for task B. 'CS-RS' means the score for content short summaries minus the score for readability short summaries. 'CL-RL' is computed in the same way for long summaries.

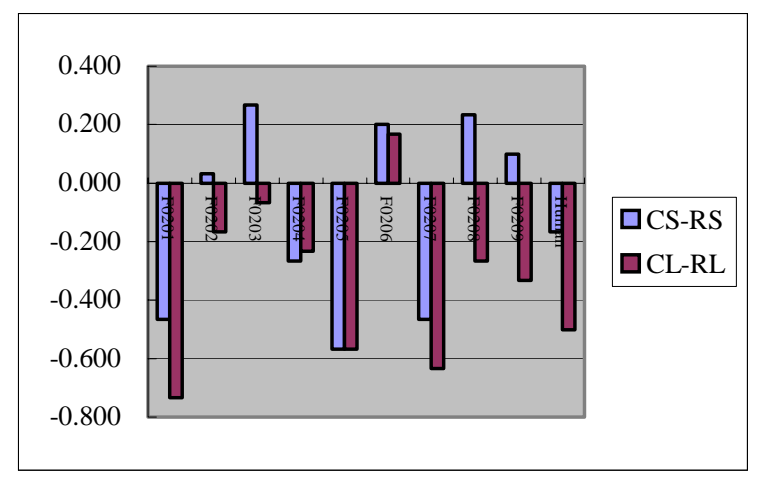

Figure 3 Score difference between content and readability (Task B)

Figure 3 shows, like Figure 1, that the scores for readability tend to be higher, thence, the differences are in minus values, than those for content for both short and long summaries. In addition, the differences are 
larger than the differences we saw for task A, i.e. in Figure 1.

Figure 4 shows the differences in scores for the different summarization lengths, i.e. short and long summaries of task B. 'CS-CL' means the score for content short summaries minus the score for content long summaries. 'RS-RL' means the score for readability short summaries minus the score for readability long summaries.

Figure 4 tells us, unlike Figure2, the scores for short summaries tend to be lower than those for long summaries. This tendency is very clear for the readability ranking scores.

Figure 1 and 3 show that when we compare the ranking scores for content and readability summaries, the readability scores tend to be higher than those for content, which means that the evaluation for readability is worse than that for content. Figure 2 and 4 shows contradicting tendencies. Figure 2 indicates that short (20\%) summaries are higher in ranking scores, i.e. worse in evaluation. However, Figure 4 indicates the other way round.

Intuitively longer summaries can have better readability since they have more words to deal with, and it is shown in Figure2. However, it is not the case with task B ranking results. Longer summaries had worse scores, especially in readability evaluation.

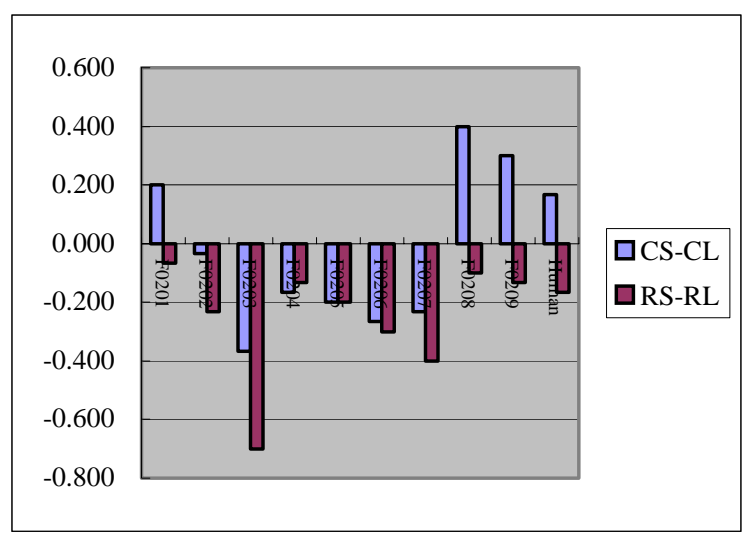

Figure 4 Score difference between different summarization lengths (Task B)

\subsection{Discussion for Evaluation by revision}

To determine the plausibility of the judges' revision, the revised summaries were again evaluated with the evaluation methods in section 5. As Tables 6 to 9 show, the degree of the revisions for the revised summaries is rather smaller than that for the original ones and is almost same as that for human summaries.

Tables 10 and 11 show the results of evaluation by ranking for the revised summaries at task $\mathrm{A}$ and $\mathrm{B}$ respectively. Compared with Tables 2 to 5, Tables 10 and 11 show that the scores for the revised summaries are rather smaller than those for the original ones and are almost same as those for human summaries.

From these results, the quality of the revised summaries is considered as same as that of human summaries.

\begin{tabular}{|c|c|c|c|c|}
\hline System No & $\begin{array}{c}\text { Content } \\
20 \%\end{array}$ & $\begin{array}{c}\text { Read- } \\
\text { ability } \\
20 \%\end{array}$ & $\begin{array}{c}\text { Content } \\
40 \%\end{array}$ & $\begin{array}{c}\text { Read- } \\
\text { ability } \\
40 \%\end{array}$ \\
\hline edit & 2.37 & 2.43 & 2.33 & 2.33 \\
\hline
\end{tabular}

Table 10 Ranking evaluation (task A)

\begin{tabular}{|c|c|c|c|c|}
\hline System No & $\begin{array}{c}\text { Content } \\
\text { Short }\end{array}$ & $\begin{array}{c}\text { Read- } \\
\text { ability } \\
\text { Short }\end{array}$ & $\begin{array}{c}\text { Content } \\
\text { Long }\end{array}$ & $\begin{array}{c}\text { Read- } \\
\text { ability } \\
\text { Long }\end{array}$ \\
\hline edit & 1.93 & 2.23 & 2.13 & 2.50 \\
\hline
\end{tabular}

Table 11 Ranking evaluation (task B)

\section{Conclusions}

We have described the outline of the Text Summarization Challenge 2. In addition to the two evaluation runs, we held two round-table discussions, one right after dryrun, and the other after formal run. At the second round-table discussion, it was pointed out that we might need to examine more closely the results of evaluation, especially the one by ranking.

We are now starting the third evaluation (TSC3). Please see our web page[4] for the details of the task.

\section{References}

[1] Proceedings of The Tipster Text Program Phase III, Morgan Kaufmann, 1999.

[2] Mani, I., et al. The TIPSTER SUMMAC Text Summarization Evaluation, Technical Report, MTR 98W0000138, The MITRE Corp., 1998.

[3] http://www-nlpir.nist.gov/projects/duc/.

[4] http://oku-gw.pi.titech.ac.jp/tsc/index-en.html.

[5] Takahiro Fukusima and Manabu Okumura, "Text Summarization Challenge -Text Summarization Evaluation at NTCIR Workshop 2", In Proceedings of NTCIR Workshop 2, pp.45-50, 2001.

[6] Takahiro Fukusima and Manabu Okumura, "Text Summarization Challenge - Text Summarization Evaluation in Japan", North American Association for Computational Linguistics (NAACL2001), Workshop on Automatic Summarization, pp.51-59, 2001.

[7] Gees C. Stein, Tomek Strazalkowski and G. Bowden Wise, "Summarizing Multiple Documents using Text Extraction and Interactive Clustering", Pacific Association for Computational Linguistics, pp.200$208,1999$. 


\section{Appendix A}

\begin{tabular}{|l|c|c|c|}
\hline $\begin{array}{l}20 \% \\
\text { readability }\end{array}$ & lead & human & humans \\
\hline F101 & 0.767 & 0.100 & 0.033 \\
\hline F102 & 0.667 & 0.100 & 0.033 \\
\hline F103 & 0.667 & 0.100 & 0.033 \\
\hline F104 & 0.733 & 0.133 & 0.067 \\
\hline F105 & 0.833 & 0.233 & 0.100 \\
\hline F106 & 0.867 & 0.233 & 0.133 \\
\hline F107 & 0.733 & 0.233 & 0.200 \\
\hline F108 & 0.833 & 0.067 & 0.033 \\
\hline human & 0.933 & 0.467 & 0.233 \\
\hline tf & 0.267 & 0.067 & 0.067 \\
\hline
\end{tabular}

\begin{tabular}{|l|c|c|c|}
\hline $\begin{array}{l}20 \% \\
\text { content }\end{array}$ & lead & human & humans \\
\hline F101 & 0.867 & 0.200 & 0.167 \\
\hline F102 & 0.900 & 0.200 & 0.100 \\
\hline F103 & 0.800 & 0.067 & 0.033 \\
\hline F104 & 0.767 & 0.067 & 0.033 \\
\hline F105 & 0.933 & 0.200 & 0.067 \\
\hline F106 & 0.900 & 0.200 & 0.100 \\
\hline F107 & 0.800 & 0.167 & 0.133 \\
\hline F108 & 1.000 & 0.267 & 0.167 \\
\hline human & 1.000 & 0.400 & 0.233 \\
\hline tf & 0.233 & 0.000 & 0.000 \\
\hline
\end{tabular}

\begin{tabular}{|l|c|c|c|}
\hline $\begin{array}{l}40 \% \\
\text { readability }\end{array}$ & lead & human & humans \\
\hline F101 & 0.833 & 0.233 & 0.033 \\
\hline F102 & 0.700 & 0.133 & 0.100 \\
\hline F103 & 0.800 & 0.100 & 0.067 \\
\hline F104 & 0.800 & 0.133 & 0.033 \\
\hline F105 & 0.767 & 0.200 & 0.167 \\
\hline F106 & 0.800 & 0.167 & 0.100 \\
\hline F107 & 0.767 & 0.200 & 0.167 \\
\hline F108 & 0.833 & 0.100 & 0.067 \\
\hline human & 0.867 & 0.467 & 0.300 \\
\hline tf & 0.400 & 0.100 & 0.100 \\
\hline
\end{tabular}

\begin{tabular}{|l|c|c|c|}
\hline $\begin{array}{l}40 \% \\
\text { content }\end{array}$ & lead & human & humans \\
\hline F101 & 0.967 & 0.167 & 0.100 \\
\hline F102 & 0.900 & 0.267 & 0.200 \\
\hline
\end{tabular}

\begin{tabular}{|l|l|l|l|} 
F103 & 0.800 & 0.100 & 0.033 \\
\hline F104 & 0.900 & 0.133 & 0.067 \\
\hline F105 & 0.867 & 0.200 & 0.167 \\
\hline F106 & 0.967 & 0.200 & 0.100 \\
\hline F107 & 0.933 & 0.233 & 0.167 \\
\hline F108 & 1.000 & 0.167 & 0.100 \\
\hline human & 0.967 & 0.300 & 0.267 \\
\hline tf & 0.367 & 0.000 & 0.000 \\
\hline
\end{tabular}

\section{Appendix B}

\begin{tabular}{|c|c|c|c|}
\hline $\begin{array}{l}\text { short } \\
\text { readability }\end{array}$ & lead & stein & human \\
\hline F201 & 0.233 & 0.167 & 0.333 \\
\hline F202 & 0.333 & 0.267 & 0.367 \\
\hline F203 & 0.367 & 0.333 & 0.533 \\
\hline F204 & 0.300 & 0.233 & 0.300 \\
\hline F205 & 0.200 & 0.233 & 0.267 \\
\hline F206 & 0.267 & 0.233 & 0.233 \\
\hline F207 & 0.200 & 0.267 & 0.400 \\
\hline F208 & 0.367 & 0.300 & 0.233 \\
\hline F209 & 0.433 & 0.167 & 0.433 \\
\hline human & 0.667 & 0.600 & 0.533 \\
\hline
\end{tabular}

\begin{tabular}{|l|c|c|c|}
\hline $\begin{array}{l}\text { short } \\
\text { content }\end{array}$ & lead & stein & human \\
\hline F201 & 0.533 & 0.400 & 0.267 \\
\hline F202 & 0.433 & 0.333 & 0.200 \\
\hline F203 & 0.500 & 0.500 & 0.100 \\
\hline F204 & 0.433 & 0.400 & 0.200 \\
\hline F205 & 0.500 & 0.533 & 0.233 \\
\hline F206 & 0.300 & 0.200 & 0.100 \\
\hline F207 & 0.633 & 0.633 & 0.233 \\
\hline F208 & 0.400 & 0.333 & 0.133 \\
\hline F209 & 0.433 & 0.267 & 0.167 \\
\hline human & 0.700 & 0.700 & 0.467 \\
\hline
\end{tabular}

\begin{tabular}{|l|c|c|c|}
\hline $\begin{array}{l}\text { long } \\
\text { readability }\end{array}$ & lead & stein & human \\
\hline F201 & 0.167 & 0.167 & 0.267 \\
\hline F202 & 0.367 & 0.333 & 0.300 \\
\hline F203 & 0.300 & 0.267 & 0.367 \\
\hline F204 & 0.233 & 0.267 & 0.333 \\
\hline F205 & 0.300 & 0.100 & 0.233 \\
\hline F206 & 0.133 & 0.100 & 0.233 \\
\hline F207 & 0.200 & 0.233 & 0.200 \\
\hline
\end{tabular}




\begin{tabular}{|l|c|c|c|} 
F208 & 0.333 & 0.300 & 0.333 \\
\hline F209 & 0.267 & 0.300 & 0.367 \\
\hline human & 0.567 & 0.533 & 0.467 \\
\hline
\end{tabular}

\begin{tabular}{|l|c|c|c|}
\hline $\begin{array}{l}\text { long } \\
\text { content }\end{array}$ & lead & stein & human \\
\hline F201 & 0.500 & 0.500 & 0.400 \\
\hline F202 & 0.533 & 0.300 & 0.167 \\
\hline F203 & 0.433 & 0.300 & 0.100 \\
\hline F204 & 0.333 & 0.400 & 0.233 \\
\hline F205 & 0.567 & 0.367 & 0.300 \\
\hline F206 & 0.200 & 0.067 & 0.167 \\
\hline F207 & 0.567 & 0.500 & 0.233 \\
\hline F208 & 0.433 & 0.533 & 0.200 \\
\hline F209 & 0.567 & 0.533 & 0.267 \\
\hline human & 0.733 & 0.700 & 0.567 \\
\hline
\end{tabular}

